\title{
Reports
}

\section{Adaptation and validation of DNA synthesis detection by fluorescent dye derivatization for high-throughput screening}

\author{
Max V. Ranall, Brian G. Gabrielli, and Thomas J. Gonda \\ University of Queensland Diamantina Institute for Cancer, Immunology and Metabolic Medicine, Princess \\ Alexandra Hospital, Brisbane, Australia
}

BioTechniques 48:379-386 (May 2010) doi 10.2144/000113410

Keywords: cell-based assay; ethynyl deoxyuridine; CuAAC; click; high-content; high-throughput; $S$ phase; DNA synthesis; proliferation

Cellular proliferation is fundamental to organism development, tissue renewal, and diverse disease states such as cancer. In vitro measurement of proliferation by high-throughput screening allows rapid characterization of the effects of small-molecule or genetic treatments on primary and established cell lines. Current assays that directly measure the cell cycle are not amenable to high-throughput processing and analysis. Here we report the adaptation of the chemical method for detecting DNA synthesis by 5 -ethynyl-2'-deoxyuridine (EdU) incorporation into both highthroughput liquid handling and high-content imaging analysis. We demonstrate that chemical detection of EdU incorporation is effective for high-resolution analysis and quantitation of DNA synthesis by high-content imaging. To validate this assay platform we used treatments of MCF10A cells with media supplements and pharmacological inhibitors that are known to affect cell proliferation. Treatments with specific kinase inhibitors indicate that EGF and serum stimulation employs both the mitogen extracellular kinase (MEK)/extracellular-regulated kinase (ERK) and phosphoinositol-3 kinase (PI3K)/AKT signaling networks. As described here, this method is fast, reliable, and inexpensive and yields robust data that can be easily interpreted.

\section{Introduction}

High-throughput screening of small molecule libraries, genetic reagents, and antibodies has been an indispensable tool for deciphering the mechanisms of disease and identifying potential therapeutics. Many diseases affect cell growth and division; therefore, assays of cell proliferation are especially important for highthroughput screening. High-throughput applications frequently employ end point assays that measure cell number by net metabolic activity $(1,2)$, ATP content (3), or biomass (4). These whole-well assays can be accurate in their reflection of cell number but do not directly measure any aspect of the cell cycle and cannot distinguish reduced growth rates from increased death. Since DNA replication in the $S$ phase of the cell cycle is a prerequisite for mitosis, proliferative capacity can be inferred from DNA synthesis through measurements of labeled nucleotide incorporation.

Cells replicating their genomes readily incorporate either radioactive or nonra- dioactive nucleoside analogs into their nascent DNA. Autoradiography of cells labeled with $\left[{ }^{3} \mathrm{H}\right]$-thymidine provided early insights into the dynamics of DNA synthesis and cell proliferation in vivo $(5-7)$. Labeling cultured cells with $\left[{ }^{3} \mathrm{H}\right]$-thymidine allowed measurement of whole populations by scintillation counting (8), making this a rapid and accurate assessment of proliferative capacity of homogeneous cell populations. The thymidine analog 5-bromo-2'-deoxyuridine (BrdU) is a nonradioactive nucleoside that can be used to measure DNA synthesis on a per-cell basis when detected by microscopy or flow cytometry (9), allowing analysis of heterogeneous cell populations. Detection of incorporated BrdU requires both a monoclonal antibody and antigen retrieval by acid (9), heat (10), or nuclease treatment $(11,12)$. Both immunodetection and enzymatic antigen retrieval are costly, while acid treatment can compromise other antigens, cell morphology, and reporter gene (e.g., GFP) function. A recently described chemical method of labeling
DNA synthesis in cells and tissues uses the alkyne-substituted nucleoside 5-ethynyl$2^{\prime}$-deoxyuridine $(\mathrm{EdU})$ as a reactive substrate for azide-substituted fluorescent dyes. During the copper-catalyzed alkyne azide cycloaddition (CuAAC or 'click') reaction, a covalent bond is formed between the alkyne base and the azide dye. This reaction is rapid, specific, and requires minimal sample processing (13). We adapted this method without the use of commercial kits or custom reagents for use with 96-well plates on a high-throughput liquid handling platform. All experimental steps were automated, and the samples were processed in situ for analysis by automated quantitative fluorescence microscopy.

We chose MCF10A non-tumorigenic mammary epithelial cells as our model system because their proliferation can be manipulated by epidermal growth factor (EGF) and serum media supplements (14-17). We combined the chemical assay of DNA synthesis with measurement of DNA content to provide a more comprehensive cell cycle analysis. 4,6'-diamidino- 

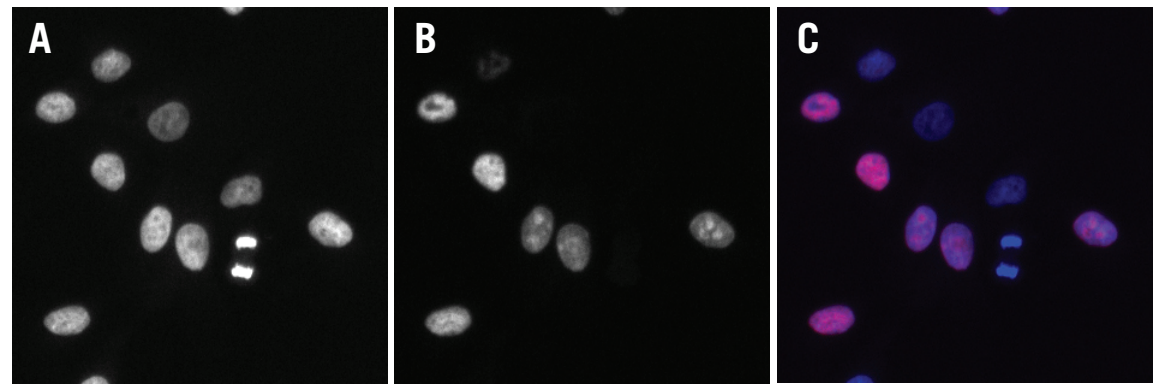

Figure 1. Fluorescence micrograph of DAPI-stained nuclei with the Cy5 adduct of the incorporated nucleoside analog EdU. MCF10A cells growing in complete medium were labeled with a 2-h pulse of $10 \mu \mathrm{M}$ EdU immediately prior to fixation. Fluorescent dye was covalently bound to the incorporated label using the CuAAC reaction to give Cy5-EdU. Images were collected with a Cellomics ArrayScan using the $20 \times$ objective and high-resolution CCD camera settings. DAPI-stained nuclei (A) and Cy5EdU (B) are pseudocolored blue and red, respectively, in the composite (C).

2-phenylindole (DAPI) is a quantitative DNA stain that can be used to resolve the $G_{0} / G_{1}, S$, and $G_{2} / M$ phases of the cell cycle (18) and has been used previously to monitor cell cycle with highcontent imaging $(19,20)$. Here we report high-content imaging analysis of DNA content and DNA synthesis by chemical detection in MCF10A cells grown in varied serum conditions, either in the presence or absence of epidermal growth factor (EGF). Similar analyses were conducted after treatments with inhib- itors that target kinases downstream of EGF: EGF receptor (EGFR), mitogen extracellular kinase (MEK), and phosphoinositol-3 kinase (PI3K). We found that the CuAAC reaction with incorporated EdU produced intensely fluorescent nuclei with DNA content intermediate to the $G_{0} / G_{1}$ and $G_{2} / M$ peaks. In these cells, DNA synthesis is dependent on EGF and, to a lesser extent, serum. Interrogation of intracellular signaling networks with kinase inhibitors in complete medium demonstrated that DNA synthesis requires
MEK signaling and is partially dependent on PI3K signaling. Z'-factor analyses-a statistical calculation of assay uniformity and dynamic range-indicate this to be a robust high-throughput assay for quantifying the S-phase population. Together these data demonstrate the successful application of this new and efficient method of detecting DNA synthesis to highthroughput and high-content screening platforms.

\section{Materials and methods}

\section{Cell culture and treatments}

Unless otherwise noted, all reagents were obtained from Sigma-Aldrich (Sydney, Australia). Experiments were performed in black, clear-bottomed 96-well tissue culture plates (Viewplates, Perkin Elmer, Waltham, MA, USA). Liquid handling steps were performed with a SciClone ALH3000 (Caliper Life Sciences, Hopkinton, MA, USA), washing steps were performed with an ELx405 plate washer (BioTek Instruments, Winooski VT, USA), and bulk dispensing steps were performed with a Wellmate (Thermo Fisher Scientific, Waltham, MA, USA).

MCF10A cells (ATCC, Manassas, VA, USA) were maintained under standard

\section{BD Flow Cytometry Systems}
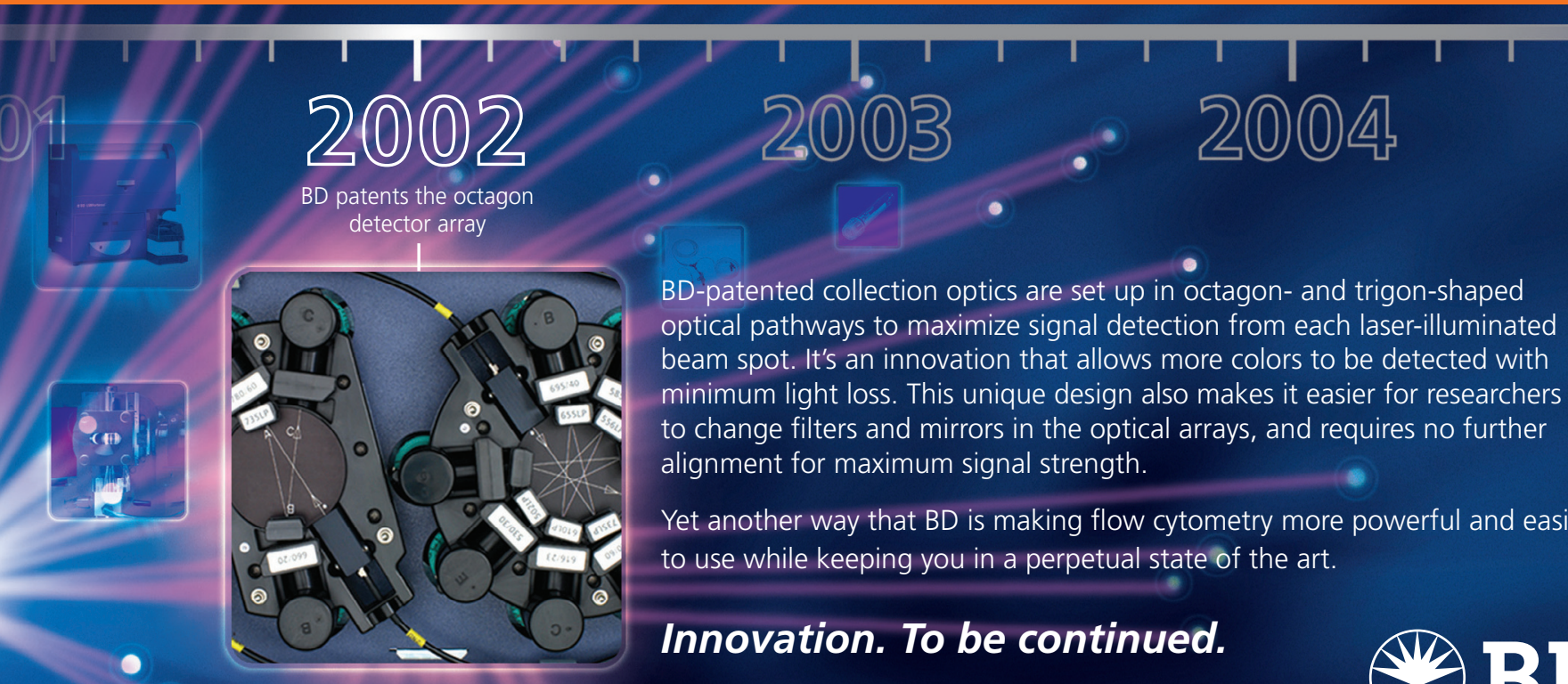

Yet another way that BD is making flow cytometry more powerful and easier to use while keeping you in a perpetual state of the art.

\section{Innovation. To be continued.}


A

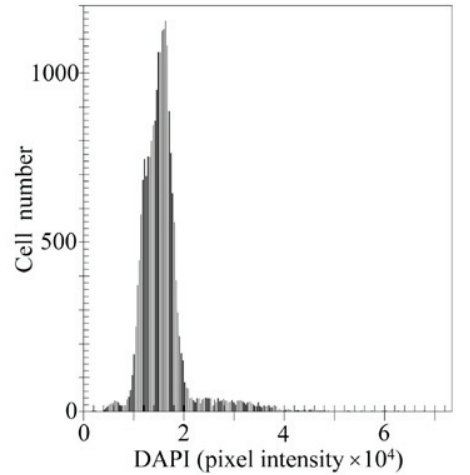

C

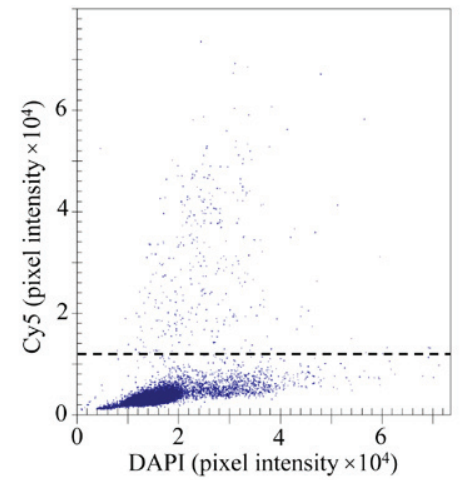

B

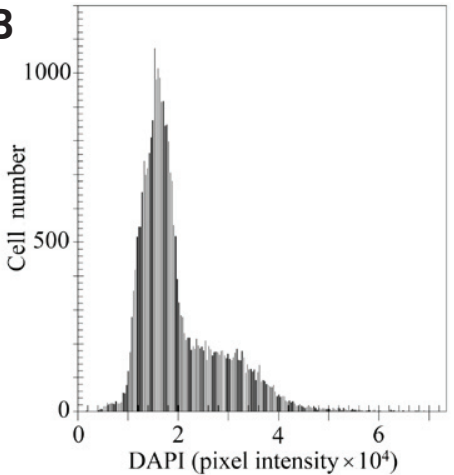

D

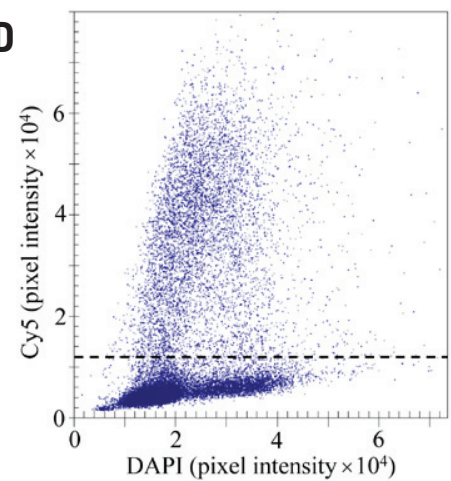

$\mathbf{E}$

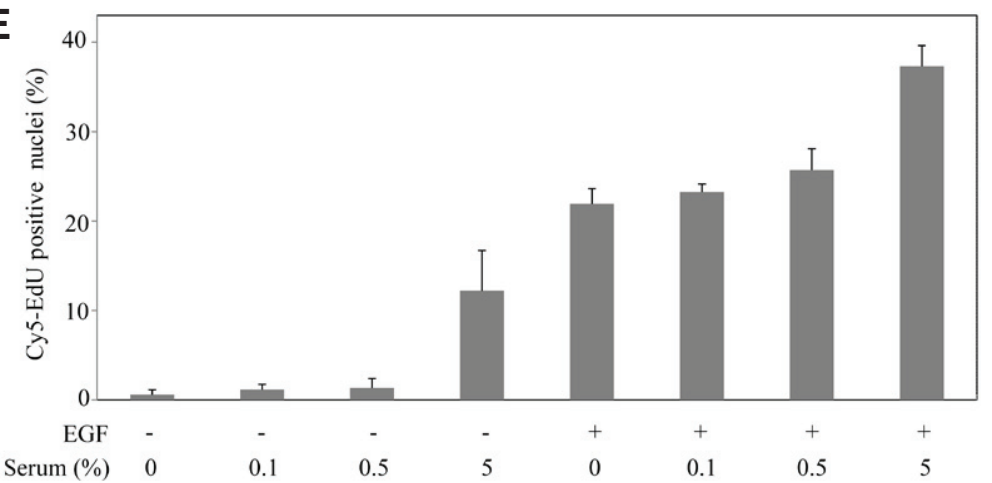

Figure 2. The effects of EGF and serum starvation on DNA content and EdU incorporation in MCF10A cells. Complete medium was withdrawn from MCF10A cells and replaced with medium containing $0 \%, 0.1 \%, 0.5 \%$, or $5 \%$ serum in the presence or absence of EGF $(20 \mathrm{ng} / \mathrm{mL})$. After $48 \mathrm{~h}$ in treatment media, cells were labeled with EdU and fixed. Samples were reacted with Cy5-azide using the CuAAC reaction, stained with DAPI, and analyzed by high-content imaging for total nuclear pixel intensity of DAPI and Cy5-EdU. Top panels show the frequency distribution of nuclear DAPI pixel intensity of cells treated with serum-free medium in the absence (A) or presence (B) of EGF. Middle panels show nuclear Cy5-EdU pixel intensity-expressed as a function of DAPI pixel intensity - of the same cells treated with serum-free medium in the absence (C) or presence (D) of EGF. A linear threshold for Cy5-EdU pixel intensity $\left(1.2 \times 10^{4}\right.$; dashed line in panels $C$ and D) was used to quantify the sub-population of MCF10A cells with high levels of Cy5-EdU after serum and EGF treatments (E). Each treatment was analyzed in four independent wells, sampling at least 5000 cells per well. Error bars $=95 \%$ confidence interval.

tissue culture conditions in 1:1 DMEM/ F12 (Invitrogen, Carlsbad, CA, USA) supplemented with $5 \%(\mathrm{v} / \mathrm{v})$ heat-inactivated horse serum (Invitrogen), $10 \mu \mathrm{g} / \mathrm{mL}$ insulin, $20 \mathrm{ng} / \mathrm{mL}$ EGF, $0.5 \mu \mathrm{g} / \mathrm{mL}$ hydrocortisone (Bayer, Leverkusen, Germany), $100 \mathrm{ng} / \mathrm{mL}$ cholera toxin, and penicillin/streptomycin antibiotic (Invitrogen). Cells were routinely passaged every 3-4 days at plating densities of $6000-7000$ cells $/ \mathrm{cm}^{2}$. For growth factor and inhibitor experiments, cells were seeded in complete medium at 2000 and 4000 cells/well, respectively, $24 \mathrm{~h}$ prior to treatment. For growth factor experiments, cultures were washed twice with PBS and then grown in $0 \%, 0.1 \%, 0.5 \%$, or $5 \%$ serumcontaining media with or without EGF for 48 h. Kinase inhibitors AG1478 (Merck
KGaA, Darmstadt, Germany), U0126 and PD98059 (Cell Signaling Technologies, Danvers, MA, USA), and LY294002 (Sigma-Aldrich) were prepared in DMSO, diluted in unsupplemented DMEM/F12, and mixed directly into the complete medium cultures. Inhibitor treatments were incubated overnight prior to labeling. In all experiments, cells were pulse-labeled with $10 \mu \mathrm{M}$ EdU (Berry and Associates, Dexter, MI, USA) for $2 \mathrm{~h}$ prior to fixation.

\section{Sample preparation and CuAAC reaction}

Cultures were washed with PBS, fixed with $3.7 \%(\mathrm{v} / \mathrm{v})$ formaldehyde in PBS, washed, permeabilized with $0.5 \%(\mathrm{v} / \mathrm{v})$ Triton X-100, washed, and then blocked with 3\% (w/v) BSA in PBS with $0.1 \%$ (v/v) Tween-20 (PBST-BSA). Prior to performing the $\mathrm{CuAAC}$ reaction, all samples were washed in PBS. Plates were routinely stored for up to 1 week at $4^{\circ} \mathrm{C}$ prior to permeabilization or following the $\mathrm{CuAAC}$ reaction.

As described by Salic and Mitchison (13), the $\mathrm{CuAAC}$ reaction mixture had four components: Tris- $\mathrm{HCl} \mathrm{pH} \mathrm{8.5,} \mathrm{CuSO}_{4}$, Cy5-azide (Lumiprobes, Moscow, Russia), and freshly prepared sodium ascorbate. Upon ascorbate addition to the reaction, the mix became very unstable; maximal signalto-noise ratios were attained by keeping all reaction components separate until they were applied to the samples. Components were combined sequentially, mixing twice with each addition, and then applied to samples immediately. To facilitate multiplate processing, Tris- $\mathrm{HCl}$ and ascorbate solutions were maintained in bulk reservoirs, while $\mathrm{CuSO}_{4}$ and $\mathrm{Cy} 5$-azide were dispensed into individual clean 96-well V-bottom plates (Corning Inc, Lowell, MA, USA) that also served as mixing vessels. The final reaction component concentrations were $100 \mathrm{mM}$ Tris- $\mathrm{HCl} \mathrm{pH} 8.5$, $4 \mathrm{mM} \mathrm{CuSO}_{4}, 2 \mu \mathrm{M}$ Cy5-azide, and 50 $\mathrm{mM}$ sodium ascorbate. Immediately after preparation, the reaction mix was dispensed into the 96-well tissue culture plate from which the PBS had been aspirated to a minimal volume. Reactions were allowed to proceed for 15-20 min in the dark and were stopped by rinsing with PBS. Residual Cy 5 was destained from samples with PBS Tween (PBST)-BSA for at least $15 \mathrm{~min}$, and then DNA was stained with $400 \mathrm{nM}$ DAPI in PBST-BSA for $1 \mathrm{~h}$ at room temperature. Samples were washed with PBS and stored at $4^{\circ} \mathrm{C}$ until analysis.

High-content imaging

Plates were scanned with a Cellomics ArrayScan (Thermo Fischer Scientific) 
automated epifluorescent microscope. Micrographs were acquired with the $10 \times$ objective and XF93 filter set. Data acquisition and analysis were controlled with the Cellomics Target Activation (v.3) application software, which maintained equivalent acquisition and processing parameters within each experiment. Exposure times were adjusted empirically to $25-35 \%$ of pixel intensity saturation. Nuclei were identified with the DAPI channel using a fixed pixel intensity threshold that was adjusted manually in each experiment to precisely delimit nuclear boundaries. Nuclei were segmented using the intensity peaks method with a setting of 4 or 5 . Background correction was applied by the surface-fitting method to both DAPI and $\mathrm{Cy} 5$ within a 150 -pixel radius from each identified nucleus. Each nucleus was assayed for the total pixel intensity of DAPI and $\mathrm{Cy} 5$ fluorescence. Analysis was limited to four wells with 5000 objects per well; Cy5-EdU-positive thresholds were set empirically according to the distribution of cells lacking $\mathrm{Cy} 5$ fluorescence.

\section{Results and discussion}

Here we describe the high-throughput application of an efficient assay that detects DNA synthesis by the formation of a covalent bond between a fluorescent dye and the incorporated nucleoside analog EdU. We found the method of Salic and Mitchison for EdU detection with the CuAAC reaction (13) to be very robust and highly sensitive without using kits or custom reagents. The assay repeatedly produced an excellent signalto-noise ratio with sub-nuclear resolution of DNA synthesis (Figure 1). During our experiments, reduction of the azide dye concentration below $2 \mu \mathrm{M}$ resulted in decreased separation between the cells with and without incorporated label (data not shown). The $\mathrm{CuAAC}$ reaction mix, without the catalyst-generating ascorbate, was stable for up to $3 \mathrm{~h}$ at room temperature with only a modest decrease in total Cy5 intensity, while labeled and unlabeled nuclei could still be distinguished clearly (data not shown).

The quantitative DNA stain DAPI was used to measure DNA content of individual MCF10A cells grown for $48 \mathrm{~h}$ in serum-free medium with or without EGF (Figure 2, B and A, respectively). In the absence of EGF, cells accumulated in $\mathrm{G}_{0} / \mathrm{G}_{1}$ with a tight Gaussian distribution centered around a mean total DAPI pixel intensity of $1.6 \times 10^{4}\left(\mathrm{SD}=4.7 \times 10^{3}\right.$, $n=21,666$; Figure 2A). Cells grown with EGF showed a non-Gaussian distribution of nuclear DAPI intensities with S-phase and $\mathrm{G}_{2} / \mathrm{M}$-phase populations (total DAPI pixel intensity $\left.>2.1 \times 10^{4}\right)$ representing $30 \%$ of the nuclei $(n=23,891$; Figure $2 B)$ compared with $7 \%$ of the nuclei in cells grown without EGF $(n=21,666$; Figure 2A). DNA synthesis, detected by the incorporation of Cy5-EdU, was observed in $1.5 \%$ of cells grown in the absence of EGF (Figure 2C) but was readily detected in $23 \%$ of cells grown in the presence of EGF (Figure 2D). A threshold for Cy5bright nuclei (Figure 2, C and D, dashed line; total Cy 5 pixel intensity $>1.2 \times 10^{4}$ ) was defined to identify nuclei with high levels of incorporated label and therefore positive for DNA synthesis. This S-phase population had a mean total DAPI pixel intensity of $2.6 \times 10^{4}\left(\mathrm{sD}=8.0 \times 10^{3}\right.$, $n=5563$ ) representing DNA content intermediate to the respective $2 \mathrm{n}$ and $4 \mathrm{n}$ of $G_{0} / G_{1}$ and $G_{2} / M$. To test the contributions of serum to sustaining $S$ phase, cells were grown for $48 \mathrm{~h}$ in serum concentrations of $0 \%, 0.1 \%, 0.5 \%$, or $5 \%(\mathrm{v} / \mathrm{v})$ with or without EGF (Figure 2E). DNA synthesis was assayed by EdU incorporation and, as above, Cy5-bright nuclei were identified as being positive for $S$ phase. These data

\section{BD Flow Cytometry Systems}

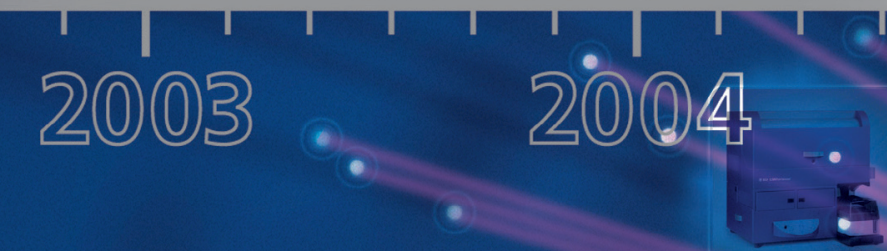

The BD special order program has supported research at the leading edge of biomedical discovery since 2005 . The custom configuration of BD flow cytometers and cell sorters provides you with a precise fit for your research and assay needs. BD's continuous and vigorous pursuit of the latest and best laser technologies ensures that the evolving needs of leading research are always met.

BD innovation gives you unparalleled choice of configuration and capabilities to keep you in a perpetual state of the art.

\section{Innovation. To be continued.}

\section{5}

BD Introduces the special order program

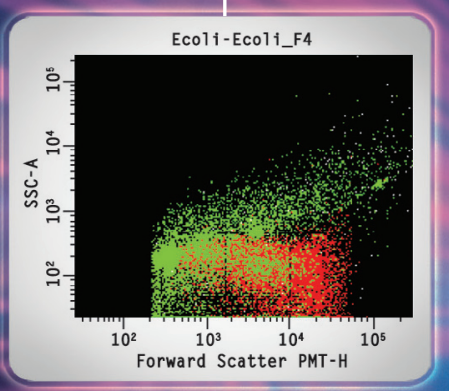

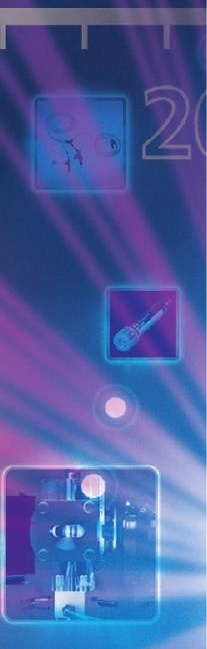


A
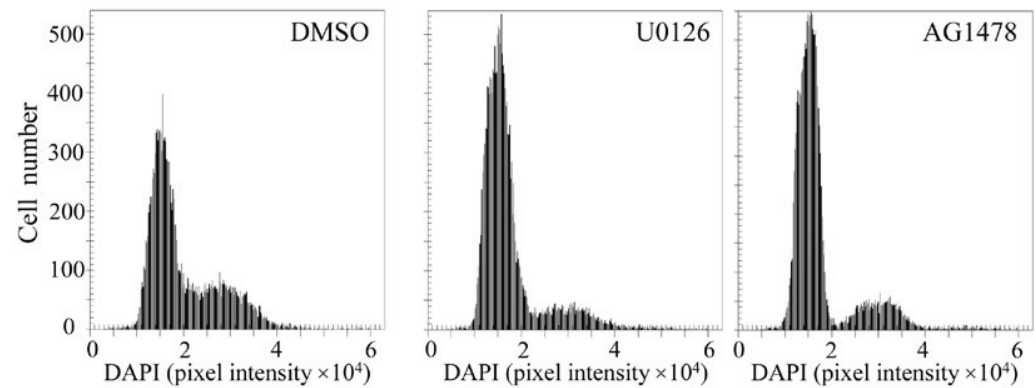

B
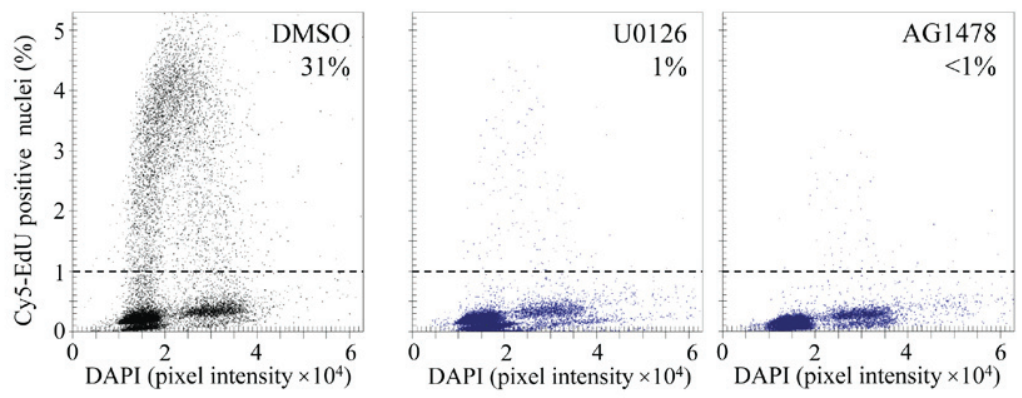

C

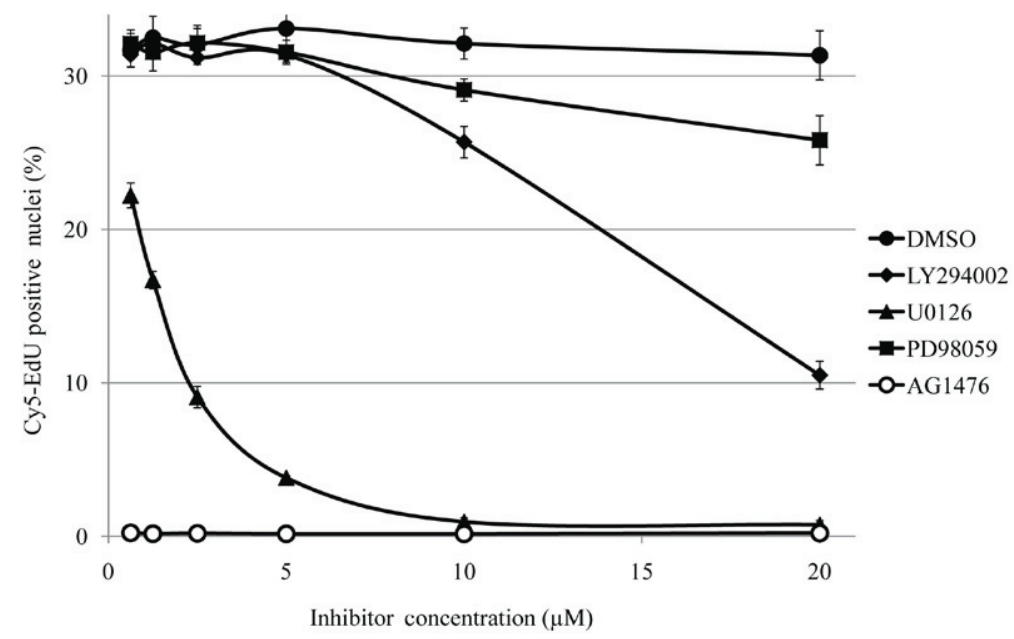

Figure 3. The effects of selected kinase inhibitors on EdU incorporation by MCF10A cells. Cells were grown in complete medium then treated overnight with a dilution series of DMSO vehicle control, EGFR tyrosine kinase inhibitor (AG1478), a potent MEK inhibitor (U0126), a weak MEK inhibitor (PD98059), or a PI3K inhibitor (LY294002). All samples were labeled with EdU and fixed. Samples were reacted with Cy5-azide using the CuAAC reaction, stained with DAPI, and analyzed by highcontent imaging for total nuclear pixel intensity of DAPI and Cy5-EdU. Total DAPI intensity DNAcontent histograms $(A)$ and scatter plots of total Cy5-EdU intensity as a function of total DAPI intensity (B) from samples treated with DMSO vehicles, $10 \mu \mathrm{M}$ U0126, or $0.6 \mu \mathrm{M}$ AG1478. A threshold for Cy5-EdU total pixel intensity $\left(>1.0 \times 10^{4}\right.$; dashed line in panel B) was used to define the fraction of the total population in S phase. This criteria was used to analyze the three-fold dilution series that treated cells with inhibitor concentrations from $0.6 \mu \mathrm{M}$ to $20 \mu \mathrm{M}$ (C). Each treatment represents the mean of four independent wells, analyzing a minimum of 3206 cells per well and 15,896 per treatment. Error bars $=95 \%$ confidence interval.

show that EGF is a major contributor to the maintenance of S phase in MCF10A cells, although both EGF and complete 5\% serum supplements were required to reach maximal rates of DNA synthesis.

To examine the intracellular signaling pathways required for progression into $S$ phase, we used inhibitors of EGFR (AG1478), MEK (U0126 and PD98059), and PI3K (LY294002). MCF10A cells grown in complete medium were treated for $16 \mathrm{~h}$ with a dilution series of each inhibitor or DMSO vehicle control, and labeled with EdU as before. DNA content analysis showed effective cell cycle arrest in $\mathrm{G}_{0} / \mathrm{G}_{1}$ by inhibition of EGFR or MEK with $0.6 \mu \mathrm{M}$ AG1478 or $10 \mu \mathrm{M}$ U0126, respectively (Figure $3 \mathrm{~A}$ ). The decrease in the $S$-phase and $\mathrm{G}_{2} / \mathrm{M}$-phase populations observed by DNA content analysis is reflected in the EdU incorporation assay: these concentrations of AG1478 or U0126 reduced Cy 5 -EdU-positive cells by $>97 \%$ relative to DMSO vehicle control cells (Figure 3B). The dilution series indicates AG1478 was the most potent inhibitor of DNA synthesis, efficiently suppressing EdU incorporation at all concentrations tested. U0126, the next most potent inhibitor, had an $\mathrm{IC}_{50}$ of $\sim 2 \mu \mathrm{M}$ in this assay context. Inhibition of PI3K by LY294002 showed less dramatic reductions in DNA synthesis with an $~ 19 \%$ reduction in the $S$-phase population at $10 \mu \mathrm{M}$ and an $\sim 42 \%$ reduction at $20 \mu \mathrm{M}$ relative to the DMSO vehicle controls (Figure 3C). The less effective MEK inhibitor, PD98059, showed only a modest effect at $20 \mu \mathrm{M}$ (Figure 3C). The discrepancy between the two MEK inhibitors is not surprising, since the described in vitro $\mathrm{IC}_{50}$ of U0126 is $\sim 0.07 \mu \mathrm{M}(\mathrm{MEK} 1 / 2)$, whereas the $\mathrm{IC}_{50}$ of PD98059 is $\sim 5 \mu \mathrm{M}$ for MEK1 and $\sim 50$ $\mu \mathrm{M}$ for MEK2 (21). These data indicate that EGFR and MEK activity are required for the maintenance of S phase while PI3K contributes substantially to this process.

Our quantitative assessment of $S$ phase by EdU incorporation is consistent with previous studies of the requirements of EGF for MCF10A proliferation (14-17). $\left[{ }^{3} \mathrm{H}\right]$-thymidine incorporation studies demonstrated that EGF is not the sole contributor to the proliferative capacity of MCF10A and that both serum and EGF are needed to reach the highest proliferation rates (16). In a survey of breastderived cell lines, MCF10A cells treated with LY294002 or U0126 in complete medium showed reduced proliferation by indirect measurement of cell number at multiple time points (17). The recapitulation of previous studies combined with the uniformity between DNA content and DNA synthesis data validate both this method of detecting DNA synthesis and the high-content imaging analysis platform. This does not, however, determine the suitability of these methods for screening applications.

To determine the robustness and sensitivity of this assay in the high-throughput screening context, we calculated the assay $Z^{\prime}$-factor as described elsewhere (22). Briefly, a $Z^{\prime}$-factor of $0.1<Z^{\prime}<0.5$ indicates a 'good' high-throughput screening assay while a $Z^{\prime}$-factor of $0.5<Z^{\prime}<1$ indicates an 'excellent' assay. For the calculation here, we used the opposing treatments (e.g., with and without EGF) as positive and negative controls. $Z$ '-factors calculated from the EGF experiments indicate that 
this assay is suitable for high-throughput screening at all three low-serum conditions: $0 \%$ serum, $Z^{\prime}=0.63 ; 0.5 \%$ serum, $Z^{\prime}$ $=0.77$; and $1 \%$ serum, $Z^{\prime}=0.51$. Analysis of the inhibitor treatments under normal grow th conditions yielded $Z^{\prime}>0.6$ for all concentrations of U0126 and $Z^{\prime}>0.8$ for all concentrations of AG1478. In contrast, LY294002 was marginally suitable for a high-throughput screening assay at $10 \mu \mathrm{M}$ with $Z^{\prime}=0.16$ but better at $20 \mu \mathrm{M}$ with $Z^{\prime}=0.53$. Together, these $Z^{\prime}$-factor data indicate that the method described here not only provides an 'excellent' assay but can do so in various experimental contexts.

Assay miniaturization is a critical consideration for applying any method to the high-throughput screening context. We have successfully applied the labeling and chemistry described here in the 384-well format with essentially no modifications to the method. The smaller format reduces sample size, thereby reducing the statistical precision of population measurements. Assay sensitivity to sample size depends largely on the experimental context. For example, limiting the number of cells analyzed in the U0126 dilution series replicates to $\sim 1100$ reduced the assay $Z^{\prime}$-factor such that the lowest concentration $(0.6 \mu \mathrm{M})$ was no longer suitable for a high-throughput screening assay, $1.3 \mu \mathrm{M}$ and $2.5 \mu \mathrm{M}$ yielded $Z^{\prime}=0.3$, while $5 \mu \mathrm{M}, 10 \mu \mathrm{M}$, and $20 \mu \mathrm{M}$ treatments retained $Z^{\prime}>0.5$. Further reduction of the sample size to $\sim 600$ cells eliminated all but the highest U0126 treatments with $Z^{\prime}=0.3$ for $5 \mu \mathrm{M}$ and $Z^{\prime}=0.8$ for $10 \mu \mathrm{M}$ and $20 \mu \mathrm{M}$. From this data, we can speculate that a screen for escape from cell cycle arrest could be robustly assayed with a minimum of 500 cells, while detection of subtle changes in $S$ phase or analysis of heterogeneous cells (e.g., transfections) would require a substantially larger sample size. Whatever the experimental context, the chemical method for DNA synthesis detection is highly specific, robust, sensitive, and very inexpensive ( $<$ \$5 USD per plate), making this high-throughput assay an attractive method for high-content screening campaigns of small-molecule and genomic libraries.

\section{Acknowledgments}

The authors would like to thank Dubravka Skalamera, Benjamin Wilson, Diwakar Ram Pattabiraman, and Paul Leo for their valued input and intellectual support. This work was supported in part by grants from the National Health and Medical Research Council (Australia) and the University of Queensland.

\section{Competing interests}

The authors declare no competing interests.

\section{References}

1. Mosmann, T. 1983. Rapid colorimetric assay for cellular growth and survival: application to proliferation and cytotoxicity assays. J. Immunol. Methods 65:55-63.

2.O'Brien, J., I. Wilson, T. Orton, and F. Pognan. 2000. Investigation of the Alamar Blue (resazurin) fluorescent dye for the assessment of mammalian cell cytotoxicity. Eur. J. Biochem. 267:5421-5426.

3. Turman, M.A. and A. Mathews. 1996. A simple luciferase assay to measure ATP levels in small numbers of cells using a fluorescent plate reader. In Vitro Cell. Dev. Biol. Anim. 32:1-4.

4.Scragg, M.A. and L.R. Ferreira. 1991. Evaluation of different staining procedures for the quantification of fibroblasts cultured in 96-well plates. Anal. Biochem. 198:80-85.

5. Taylor, J.H., P.S. Woods, and W.L. Hughes. 1957. The organization and duplication of chromosomes as revealed by autoradiographic studies using tritium-labeled thymidine. Proc. Natl. Acad. Sci. USA 43:122-128.

6. Gowans, J.L. 1959. The recirculation of lymphocytes from blood to lymph in the rat. J. Physiol. 146:54-69.

7. Cronkite, E.P., T.M. Fliedner, V.P. Bond, J.R. Rubini, G. Brecher, and H. Quastler. 1959. Dynamics of hemopoietic proliferation in man and mice studied by H3-thymidine incorporation. Ann. N.Y. Acad. Sci. 77:803-820.

8. Fallot, P., A. Girgis, M. Laine-Boszormenyi, and J. Vieuchange. 1965. Utilisation du compteur á scintillations en milieu liquid pour la mesure directe de la radioactivité du tritium incoporé dans des cellules de mammifères cultivée in vitro. Int. J. Appl. Radiat. Isot. 16:349-356.

9. Gratzner, H.G., R.C. Leif, and D.J. Ingram. 1975. The use of antibody specific for Bromodeoxyuridine for the immunofluorescent determination of DNA replication in single cells and chromosomes. Exp. Cell Res. 95:88-94.

10.Tang, X., D.L. Falls, X. Li, T. Lane, and M.B. Luskin. 2007. Antigenretrieval procedure for bromodeoxyuridine immunolabeling with concurrent labeling of nuclear DNA and antigens damaged by $\mathrm{HCl}$ pretreatment. J. Neurosci. 27:5837-5844.

11. Gonchoroff, N.J., J.A. Katzmann, R.M. Currie, E.L. Evans, D.W. Houck, B.C. Kline, P.R. Greipp, and M.R. Loken. 1986. S-Phase detection with an antibody to bromodeoxyuridine. Role of DNase pretreatment. J. Immunol. Methods 93:97-101.

12. Dolbeare, F. and J.W. Gray. 1988. Use of restriction endonucleases and exonucleaseIII to expose halogenated pyrimidines for immunological staining. Cytometry 9:631-635.

13. Salic, A. and T.J. Mitchison. 2008. A chemical method for fast and sensitive detection of DNA synthesis in vivo. Proc. Natl. Acad. Sci. USA 105:2415-2420.

14.Soule, H.D., T.M. Maloney, S.R. Wolman, W.D. Peterson, Jr., R. Brenz, C.M. McGrath, J. Russo, R.J. Pauley, et al. 1990. Isolation and characterization of a spontaneously immortalized human breast epithelial cell line, MCF-10. Cancer Res. 50:6075-6086.

15.Ethier, S.P., R.M. Summerfelt, K.C. Cundiff, and B.B. Asch. 1990. The influence of growth factors on the proliferative potential of normal and primary breast cancer derived human breast epithelial cells. Breast Cancer Res. Treat. 17:221-230.

16. Graham, N.A. and A.R. Asthagiri. 2004. Epidermal growth factor-mediated T-cell factor/lymphoid enhancer factor transcriptional activity is essential but not sufficient for cell cycle progression in nontransformed mammary epithelial cells. J. Biol. Chem. 279:23517-23524.

17. Ripple, M.O., S. Kalmadi, and A. Eastman. 2005. Inhibition of either phosphatidylinositol 3-kinase/AKT or the mitogen/extracellularregulated kinase, MEK/ERK, signaling pathwyas suppress growth of breast cancer cell lines, but MEK/ERK signaling is critical for cell survival. Breast Cancer Res. Treat. 93:177-188.

18. Hamada, S. and S. Fujita. 1983. DAPI staining improved for quantitative cytofluorometry. Histochem. Cell Biol. 79:219-226.

19. Barabasz, A., B. Foley, J.C. Otto, A. Scott, and J. Rice. 2006. The use of highcontent screening for the discovery and characterization of compounds that modulate mitotic index and cell cycle progression by differing mechanisms of action. Assay Drug Dev. Technol. 4:153-163.

20.Gasparri, F., P. Cappella, and A. Galvani. 2006. Multiparametric cell cycle analysis by automated microscopy. J. Biomol. Screen. 11:586-598.

21.Alessi, D.R., A. Cuenda, P. Cohen, D.T. Dudley, and A.R. Saltiel. 1995. PD 098059 is a specific inhibitor of the activation of mitogen-activated protein kinase kinase in vitro and in vivo. J. Biol. Chem. 270:2748927494.

22.Zhang, J.H., T.D.Y. Chung, and K.R. Oldenburg. 1999. A simple statistical parameter for use in the evaluation and validation of high throughput screening assays. J. Biomol. Screen. 4:67-73.

Received 9 December 2009; accepted 16 March 2010.

Address correspondence to Thomas J. Gonda, University of Queensland, Diamantina Institute for Cancer, Immunology and Metabolic Medicine, Princess Alexandra Hospital, Ipswich Rd., Brisbane QLD 4102, Australia. e-mail: t.gonda@uq.edu.au 